\title{
Using Google Adwords to Recruit Family Carers of People with Dementia
}

\author{
Siobhan T. O’Dwyer \\ Wendy Moyle \\ Centre for Health Practice Innovation \\ Griffith University
}




\begin{abstract}
As rates of home Internet access rise, online advertising may provide a new means of recruiting research participants. Google Adwords is one approach to online recruitment, but only a handful of studies have reported the cost and effectiveness and none have focused on older adults. This paper describes a Google Adwords campaign designed to recruit family carers of people with dementia to a cross-sectional study of wellbeing. The aim is to provide some insight for researchers planning to use Google Adwords to recruit other older adults. The ads were viewed more than 450,000 times in a six-week period, but only 49 surveys were completed, at a cost of $\$ 122$ per survey. Google Adwords has promise, but much remains to be understood about how to maximize its potential in ageing research. Recommendations for the design of future Google Adwords campaigns are provided.
\end{abstract}

\title{
Keywords
}

Recruitment, internet, dementia, carers 


\section{Introduction}

A failure to recruit sufficient numbers of participants is a common problem in ageing research [1], but online advertising may offer a novel solution, particularly as more than half $(54 \%)$ of all older Australians now have Internet access at home [2]. Google Adwords (GA; https://adwords.google.com.au) is one method of online advertising, but there is little to guide researchers who are interested in using it.

Adwords are the highlighted advertisements that appear above or next to Google search results. Advertisers select keywords or phrases, create an ad, and allocate a budget and timeframe for their campaign. The ads appear in response to searches that match the keywords or phrases. People who click on the ad are taken to a website nominated by the advertiser. The advantages of GA for researchers are: there is no minimum spend or timeframe; a cost is only incurred when someone clicks on the ad; and the appearance of ads can be limited by geographical region.

Only a handful of studies have reported the outcomes of GA recruitment campaigns and while some limitations have been identified (such as the low rate of conversion from ad clicks to study participation and the potential for bias if used as the only recruitment method [4,5]), the outcomes have generally been positive. Buller and colleagues [3], for example, compared GA with three other methods for recruiting young people to a smoking cessation intervention. They found it "less expensive and more effective (in terms of generating enrollees) than any other recruitment method used" (p584). Similarly, 
Morgan, Jorm and Mackinnon [4] found GA less time-consuming and more effective than other strategies for recruitment to an online depression intervention, while Temple and Brown [5] used it to recruit 477 people to an online survey of cannabis use in just 18 days. None of the existing studies, however, have focused specifically on older adults.

The aim of this paper is to describe a GA campaign used to recruit family carers of people with dementia (PWD) to a cross-sectional study. With more than half of all family carers of PWD aged over 65 [10] and one in five older adults providing care for a person with a long-term illness or disability [6], the experience might provide useful insights for other ageing researchers planning to use GA. An overview of the study is provided, along with a description of the campaign and its outcomes. The effectiveness of the campaign is compared with previous research and recommendations made for the design of future campaigns.

\section{Study Overview}

The study was a cross-sectional survey of the wellbeing of family carers of PWD, with a particular focus on suicide risk in this population. It was approved by the University Human Research Ethics Committee and had a target sample size of 1,500, spread across three groups (those caring at home, HC; those caring for a PWD living in residential care, IC; and those bereaved in the last 2 years; BD). The survey (which was completed anonymously) included demographic and caring questions, along with a number of scales relating to physical and mental health, and took approximately 30 minutes to complete. A 
slightly different version of the survey was developed for each group and the surveys were available online and in hard copy. The online surveys included a 'save and resume later' function to minimize participant burden. While all recruitment, informed consent, and survey materials framed the research as a study of physical and emotional wellbeing, all participants were informed prior to consent that the survey included questions about suicide and contact details for telephone support or crisis lines were provided prior to consent and throughout the survey. A range of recruitment methods was employed, including: newspaper advertising; promotion at community events and conferences; direct invitations via advocacy organisations and in-home and residential aged care providers; social media; radio interviews; and word of mouth. After eight months of recruitment less than 600 surveys had been completed and a decision was made to use online advertising to boost the sample size in the remaining three months allocated to recruitment.

\section{Google AdWords Campaign}

The campaign was conducted by a digital marketing agency that was given a brief to attract between 500 and 1,000 Australian family carers of PWD to the research. Based on this brief, two ads were designed and more than 800 keywords and phrases selected.

The ads for this campaign are in Figure 1. They comply with the GA format of title (max. 25 characters), two lines of description (max. 35 characters per line), and shortened website address (max. 35 characters). 
The ads were restricted to Google searches conducted in Australia. Carers who clicked on the ads were taken to the online survey's front page that contained all the information about the study (as required by the Human Research Ethics Committee), as well as researcher contact details for carers who preferred a hard copy survey. Carers were informed that completion of the survey would be taken as a sign of consent. After reading this information, carers clicked a button labeled Next to indicate consent and were taken to a page containing a single-item screening questionnaire. This was used to direct carers to the most appropriate version of the survey (HC, IC, or BD) or away from the site if they were ineligible.

The campaign ran for six weeks from early November to mid-December 2012. AUD $\$ 7,000$ was allocated to the campaign, including a management fee equivalent to $20 \%$ of the final amount spent on advertising. No other recruitment methods were employed during this period.

\section{Results}

The ads appeared 454,007 times in six weeks and were clicked on 1,508 times. The most

clicks came from people searching for the terms dementia (20 clicks), Alzheimer's (11 clicks) and dementia test (11 clicks). The remaining clicks came from nearly 900 different search terms, each resulting in between 1 and 9 clicks. Six of the top 15 search terms were associated with professional caregiving - career aged care ( 7 clicks), 
employment aged care (5 clicks), aged care courses perth (5 clicks), job aged care (4 clicks), jobs caregiver (4 clicks).

Sixty-eight people completed the screening questionnaire (one person proceeded to this page, but closed the survey before responding). Thirty-three people identified themselves as $\mathrm{HC}, 14$ as IC, and 8 as BD. Thirteen selected "I am none of the above" and were directed away from the survey. Forty-nine online surveys were completed in the six-week period (six people who completed the screening questionnaire did not complete the survey). There were five requests for hard copy surveys. Figure 1 shows the flow of responses.

Due to the small number of clicks, the budget was underspent by $\$ 1,036.86$. The advertising spend was $\$ 4969.28$, with a management fee of $\$ 993.86$. Based on the total amount spent $(\$ 5,963.14)$, the average cost per click was $\$ 3.95$, with an average cost per completed survey of $\$ 121.70$.

\section{Discussion}

The GA campaign was less successful than expected, with only 49 surveys completed during the allocated timeframe, at a cost of $\$ 122$ per survey.

Although the small number and varied design of previous studies makes comparison difficult, the proportion of clicks resulting in participation in this study $(3.25 \%)$ is within 
the range reported by others $(0.08 \%$ to $24 \%[4,7])$. The cost per participant, however, is noticeably higher. Previous studies have reported costs ranging from $\sim \$ 6$ to $\sim \$ 40[8,3]$, but, as Buller and colleagues note [3], an increased cost is to be expected in studies that target smaller, more specialized populations.

One of the main challenges associated with GA is balancing "broad exposure with appropriate targeting and tailoring of recruitment messages" [4, p.9]. In the current study the ad wording appears to have been too generic, particularly given the high number of clicks from people seeking work in aged care. Including the term family in the ads might have minimized clicks by paid care workers and boosted clicks by family carers, thereby increasing the number of clicks resulting in completed surveys.

The timeframe for the campaign also appears to have been insufficient. The marketing agency reported "a lack of relevant search volume" over the six weeks and noted that a longer timeframe would have exposed more people to the ads, potentially increasing the number of clicks and the number of surveys completed (Personal Communication, 2013). While more time does not guarantee more participants (compare [4], [5], [7], and [9]), a longer timeframe in the current study would have allowed search terms and ads to be modified over time (e.g. removing the terms attracting aged care workers), which may have resulted in a more targeted campaign and reduced costs per completed survey. 
Finally, it may be that GA is simply not an engaging recruitment method for the current cohort of carers. More than half of all family carers of PWD are aged 65 and over [10] and were not raised with this technology. They may not understand how the ads work or may be suspicious of online advertisements. Gaining the trust of potential participants is a challenge in all online recruitment $[4,5]$ and this might be particularly so for older people. While this may present an impediment to online recruitment with the current cohort of dementia family carers, and older adults more generally, it will become less of a problem as more technologically experienced generations reach later life.

Despite the limitations of this GA campaign, it still resulted in 49 completed surveys; a not insignificant contribution to the final sample. Given that the campaign came after eight months of extensive recruitment, it seems that the real strength of this approach was the ability to attract family carers who had not been reached by any other recruitment method.

GA is a novel approach to recruitment, but much remains to be understood about how to capitalize on this approach in ageing research. Future campaigns may benefit from longer timeframes, as well as monitoring and modifying keywords and ads during the campaign. As the more technologically experienced generations get older, GA might become an increasingly useful means of recruitment.

\section{Key Points}


- As rates of home Internet access reach nearly 80\%, online advertising may provide a new approach to the ongoing problem of recruiting sufficient numbers of older adults to research

- Google Adwords are a form of online advertising, but there are few reports to guide researchers

- After a six-week Google Adwords campaign, 49 family carers of people with dementia completed a survey about their wellbeing, at a cost of $\$ 122$ per participant

- In future, longer timeframes and ongoing modifications to ads and search terms might improve the outcomes when using Google Adwords to recruit older adults 


\section{References}

1. Mody L, Miller DK, McGloin JM, Freeman M, Marcantonio ER, Magaziner J, Studenski S. Recruitment and retention of older adults in aging research. JAGS. 2008; 56: 2340-2348.

2. Australian Bureau of Statistics. Household use of information technology, Australia 2010-2011 (8146.0). Canberra: ABS, 2011.

3. Buller DB, Meenan R, Severson H, Halperin A, Edwards E, Magnusson B. Comparison of 4 recruiting strategies in a smoking cessation trial. Am J Health Behav. 2012; 36: 577-588.

4. Morgan AJ, Jorm AF, Mackinnon AJ. Internet-based recruitment to a depression prevention intervention: Lessons from the Mood Memos study. $\mathrm{J}$ Med Internet Res. 2013; 15: e31.

5. Temple EC, Brown RF. Recruitment methods: Engaging the hidden population of cannabis users in research. J Res Pract. 2011; 7: D2.

6. Australian Institute of Health and Welfare. Older Australia at a glance $\left(4^{\text {th }}\right.$ Ed). Canberra: AIHW, 2007.

7. Hoffman SR, Stallings SF, Bessinger RC, Brooks GT. Difference between health and ethical vegetarians: Strength of conviction, nutrition knowledge, dietary restriction, and duration of adherence. Appetite. 2013; 65: 139-144.

8. Gordon JS, Akers L, Severson HH, Danaher BG, Boles SM. Successful participant recruitment strategies for an online smokeless tobacco cessation program. Nicotine Tob Res. 2006; 8: S35-S41. 
9. Kim AS, Poisson SN, Easton JD, Johnston SC. A cross-sectional study of individiuals seeking information on transient ischemic attack and stroke symptoms online: A target for intervention? PLos ONE. 2011; 7.

10. Australian Institute of Health and Welfare. Dementia in Australia. Canberra:

AIHW, 2012. 


\section{Figure Legends}

Figure 1. The two Google Adwords advertisements used in this campaign.

Figure 2. Flow of responses from ads to completed surveys. 